\title{
Modelos artesanais no ensino e prática da técnica cirúrgica veterinária
}

\author{
[Handmade models for surgical skills teaching and practicing]
}

\section{"Artigo Científico/Scientific Article"}

\author{
James Newton Bizetto Meira de Andrade*, Cibelly Alves Barcelos, Eric Francelino Andrade, \\ Heloisa Maria Falcão Mendes, Ricardo Andres Ramirez Uscategui, Adalfredo Rocha Lobo Júnior
}

Instituto de Ciências Agrárias (ICA), Universidade Federal dos Vales do Jequitinhonha e Mucuri (UFVJM), Unaí-MG, Brasil

*Autor para correspondência/Corresponding author: E-mail: james.andrade@ufvjm.edu.br

\begin{abstract}
Resumo
A prática cirúrgica requer técnica adequada e habilidade manual. Diversas alternativas têm sido propostas ao uso de animais no ensino prático das técnicas cirúrgicas, como cadáveres, softwares, simuladores, entre outros. O objetivo deste estudo foi desenvolver e avaliar modelos de ensino e aprendizagem em técnica cirúrgica veterinária. Estudantes de medicina veterinária confeccionaram bastidores de incisão e sutura, suportes para fixar bananas, nos quais treinariam incisões e suturas, plataformas para simulação de ligadura de vasos, simuladores de parede abdominal e de aparelho genital feminino, para treino de ovariohisterectomia (OSH). Após terem recebido conhecimento teórico, puderam praticar as manobras nos modelos, ao longo do semestre. Os alunos responderam a um questionário de 5 pontos de Likert, por meio do qual se avaliou a percepção dos mesmos em relação ao método. As plataformas desenvolvidas mostraram-se úteis na prática de incisões, suturas e ligaduras, assim como no treinamento de OSH. Houve elevada frequência de respostas positivas favoráveis ao método, bem como alta frequência de respostas negativas em oposição ao mesmo $(\mathrm{p}<0,05)$. Concluiu-se que os modelos foram efetivos no ensino e aprendizado de habilidades cirúrgicas básicas em técnica cirúrgica veterinária.
\end{abstract}

Palavras-chave: Habilidades; treinamento alternativas; cirurgia.

\begin{abstract}
Surgery requires technical skills. Several alternative methods have been proposed for animals' usage in surgery teaching practice, such as cadavers, software, surrogates, among others. The purpose of this report was to develop and evaluate surgical skill learning and practice models. Veterinary students have built by themselves incision and suture training pads, supports for holding bananas to train incision and suture patterns, platforms for training vessels ligature techniques, abdominal wall surrogates, and female genital tract models for ovariohysterectomy training. After theorical classes and practical explanations, they have practiced surgical skills along the semester on the models. A feedback questionnaire based on five-point Likert scale was applied to evaluate the perception of the students related to the method. Platforms developed were useful to practice incisions, ligature techniques, suture patterns and ovariohysterectomy training. There was a high frequency of positive answers in favor of the method, and a high frequency of negative answers for questions opposite to it $(\mathrm{p}<0.05)$. Conclusion: We conclude that the models were effective in veterinary surgical basic skills teaching and learning.
\end{abstract}

Keywords: skills; training; alternatives; surgery.

\section{Introdução}

A cirurgia é um campo que, além do conhecimento, requer habilidades técnicas e constante treinamento. Durante muito tempo seu ensino foi realizado utilizando-se animais vivos ou cadáveres. Atualmente, a vivissecção é desencorajada na grande maioria dos países (Griffon et al., 2000; Smeak, 2007). Cadáveres são 
utilizados, entretanto, possuem algumas limitações como ausência de sangramento e autólise. Esta, por sua vez pode ser reduzida por meio de utilização de conservantes, como por exemplo a solução de Larssen (Silva et al., 2004).

De acordo com Smeak (2007), classicamente espera-se dos estudantes de medicina veterinária maior capacidade em realizar cirurgias complicadas do que dos estudantes de medicina do mesmo período e que, portanto, sejam capazes de realizar cirurgias mais complicadas após se formarem do que os médicos humanos. Por exemplo, é muito raro um médico recém-formado realizar uma cirurgia de cesariana, como cirurgião, já para o veterinário, é comum. Por outro lado, os veterinários têm menos oportunidades de pósgraduação ou de aprendizado do após se graduarem do que os médicos.

O treinamento adequado de habilidades cirúrgicas ao longo da graduação, no curso de medicina veterinária, de maneira estratégica e contínua, é de grande importância na formação do cirurgião veterinário. Com relação às metodologias comumente utilizadas no ensino da técnica cirúrgica, observou-se, recentemente, que o método "procedure-oriented" (procedimento orientado), ou seja, aquele em que os alunos realizavam uma determinada técnica cirúrgica e o professor observava e os orientava se mostrou ineficaz. Isso se deveu principalmente ao fato de não ser possível se ensinar ao mesmo tempo as habilidades cirúrgicas, que são necessárias a qualquer procedimento cirúrgico, e a técnica cirúrgica em si. Foram notadas diversas falhas técnicas, o que por sua vez refletia em sofrimento dos pacientes, resultando em uma experiência negativa (Smeak, 2007).

Atualmente são preferidos os métodos "skills-oriented", ou seja, orientados por habilidades, por meio dos quais foca-se mais em um procedimento, ou em uma técnica, ou em uma habilidade. Um exemplo seria a realização de treinamento em cirurgias de $\mathrm{OSH}$, repetidas vezes, podendo-se assim praticar ligaduras, pinçamentos com gentileza, entre outras manobras. O método permite a fixação das manobras nos praticantes, permitindo sua repetição em outras cirurgias, pois o princípio é o mesmo e a técnica similar (Smeak, 2007).

Para estudantes de medicina veterinária, o fato de poder aprender e treinar habilidades cirúrgicas antes de praticar em animais vivos seria uma grande oportunidade para incrementarem seu aprendizado, suas aptidões, e sua autoconfiança. De fato, Griffon et al. (2000) relataram essa necessidade de melhora no treinamento cirúrgico em uma instituição de ensino, devido a complicações observadas. Diversos métodos alternativos, como vídeos, programas interativos, dispositivos e modelos para o treinamento de suturas, modelos de estruturas anatômicas, cadáveres conservados, peças de abatedouros, e simuladores virtuais, entre outros, têm sido propostos para o ensino e treinamento da técnica cirúrgica (Smeak, 2007; Fransson e Ragle, 2010; Hill e Smeak., 2010; Costa Neto et al., 2012; Denaday et al., 2014; Giusto et al., 2015; Fahie et al., 2016; Read et al., 2016; Williamson et al., 2019). Todos os métodos possuem suas vantagens e limitações (por exemplo, cadáveres são excelentes para se demonstrar as diferentes camadas de tecidos, porém não se pode promover sangramentos), devendo assim ser usados conjuntamente, otimizando o processo de aprendizagem e treinamento (Smeak, 2007).

$\mathrm{O}$ custo do método e a sustentabilidade também são importantes fatores a serem considerados. Muitas instituições solicitam aos estudantes que adquiram seus próprios kits de treinamento cirúrgico, de modo que materiais econômicos, preferencialmente recicláveis são benvindos.

De acordo com Griffon et al. (2000), a tendência contemporânea em relação à educação cirúrgica mundial volta-se para os modelos alternativos. No presente trabalho, objetivou-se o desenvolvimento de modelos confeccionados artesanalmente para o treinamento de manobras cirúrgicas fundamentais, como diérese, hemostasia e síntese, além da técnica específica de ovariohisterectomia. Nossa hipótese foi de que a prática das manobras cirúrgicas nos modelos poderia aumentar $\mathrm{o}$ aprendizado, melhorar gradualmente as habilidades e a confiança na realização das manobras cirúrgicas fundamentais.

\section{Material e métodos}

\section{Dispositivos desenvolvidos}

Estudantes do $7^{\circ}$ período do curso de medicina veterinária construíram artesanalmente os seguintes dispositivos para treinamento de manobras cirúrgicas: bastidores para treinamento de incisão e sutura, suportes para fixar bananas, plataformas para treinamento de ligaduras 
vasculares e simuladores de parede abdominal e do aparelho reprodutor feminino.

Os bastidores de incisão e sutura foram confeccionados com peças de $25,0 \mathrm{~cm}$ x $20,0 \mathrm{~cm}$ de espuma vinílica acetinada (EVA) coladas sobre peças de madeira ou de fibra de média densidade (MDF), oriundas de sobras de fábricas locais. Foram colocadas borrachas antiderrapantes autoadesivas em cada canto inferior (Figura 1).

Os suportes para bananas foram feitos a partir de peças de $20 \mathrm{~cm}$ de canos de 10 polegadas de cloreto de polivinila (PVC), serrados ao meio, nos quais foram feitos diversos furos, através dos quais foram colocados elásticos (Figura 2).

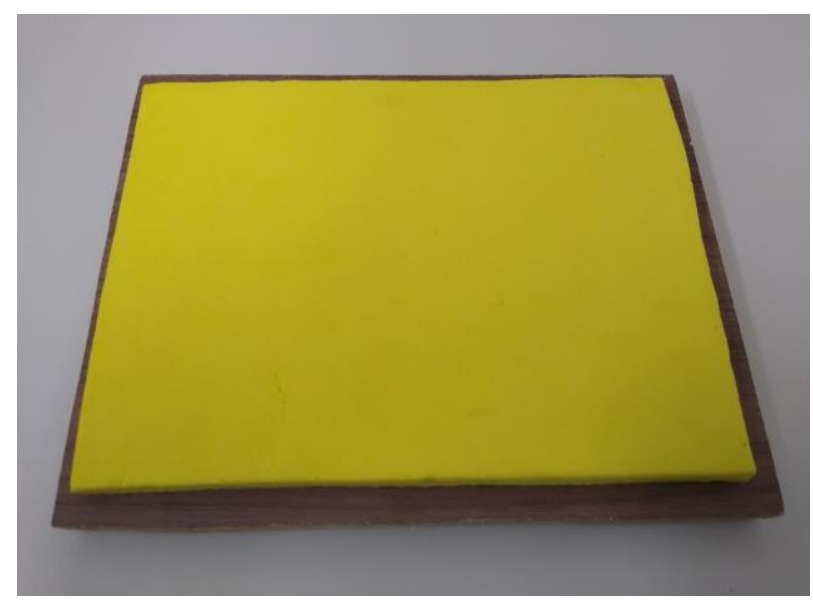

Figura 1. EVA sobre plataforma de MDF para treinamento de incisões.

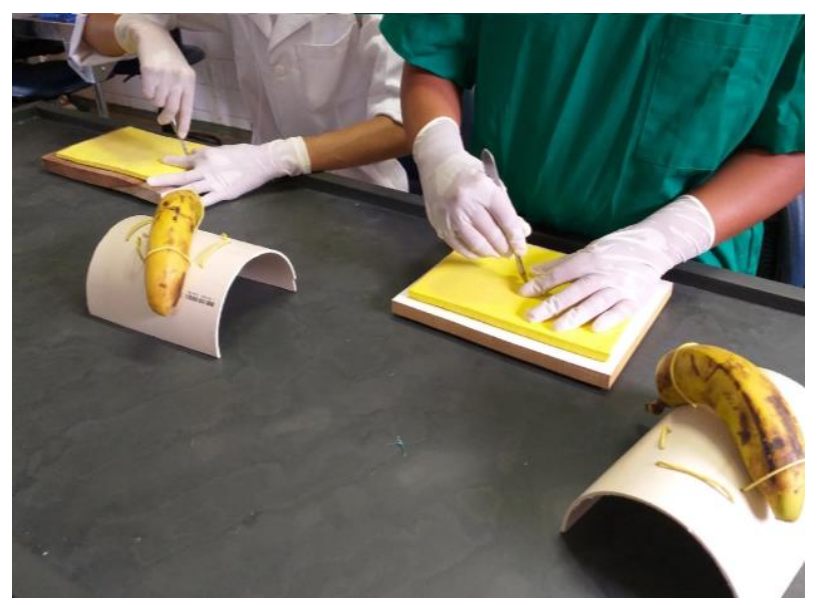

Figura 2. Plataformas de treinamento de incisão e sutura. Notar as bananas devidamente acopladas.

As plataformas para treinamento de ligaduras vasculares foram construídas colando-se três sondas de diferentes diâmetros em uma peça de madeira retangular (Figura 3).

Já o simulador de parede abdominal foi feito com três camadas de EVA, de diferentes cores, as quais representariam pele, subcutâneo e musculatura. Foram atravessadas, ortogonalmete à futura incisão, algumas sondas uretrais $n^{\circ} 6$ entre as camadas, as quais foram conectadas a seringa de 20 $\mathrm{mL}$ e preenchidas por hidróxido férrico diluído em solução fisiológica, para simular hemorragia, ao serem incisadas. Para tal, após preenchidas, a extremidade foi fechada com um nó e as seringas foram "armadas" com elásticos, estando com o êmbolo completamente puxado e a seringa, repleta (Figura 4). Ao serem incisadas as camadas e, consequentemente, as sondas, que representariam os vasos, a pressão do elástico deslocaria o líquido, simulando a hemorragia. As peças foram acopladas a cãezinhos de pelúcia, permitindo treinamento de colocação de campos e manobras de antissepsia (Figura 5).



Figura 3. Plataforma para prática de técnicas de ligaduras vasculares. Estudantes realizando ligadura por transfixação.

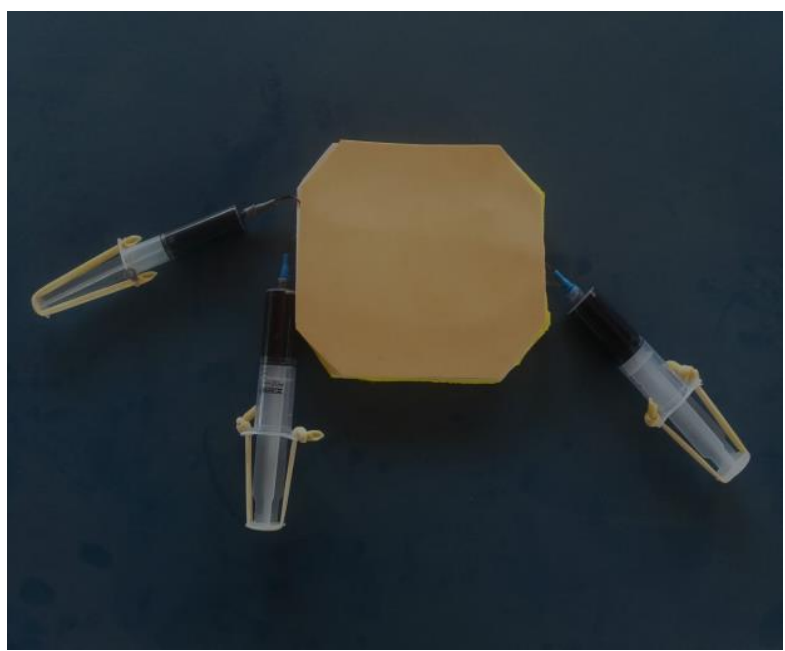

Figura 4. Simulador de parede abdominal confeccionado com três camadas de EVA. Cateteres uretrais preenchidos por hidróxido férrico foram colocados entre as camadas, perpendicularmente ao sentido das futuras incisões e acoplados a seringas, para simular hemorragias. 
O simulador do trato genital feminino foi montado com tubos de silicone, em forma de 'Y". Os ovários foram representados por duas bolinhas de isopor coladas na extremidade de cada tubo e os ligamentos, por elásticos. A peça foi colada sobre um filme de PVC, que representou os ligamentos largo e redondo. Os vasos foram simulados por meio de sondas preenchidas por hidróxido férrico. O modelo foi acoplado a meio-cano de PVC para melhor introduzir no cãozinho de pelúcia (Figura $6)$.

Os modelos foram utilizados ao longo do semestre para treinamento de diferentes manobras cirúrgicas fundamentais, como incisões, técnicas de hemostasia, suturas e OSH.

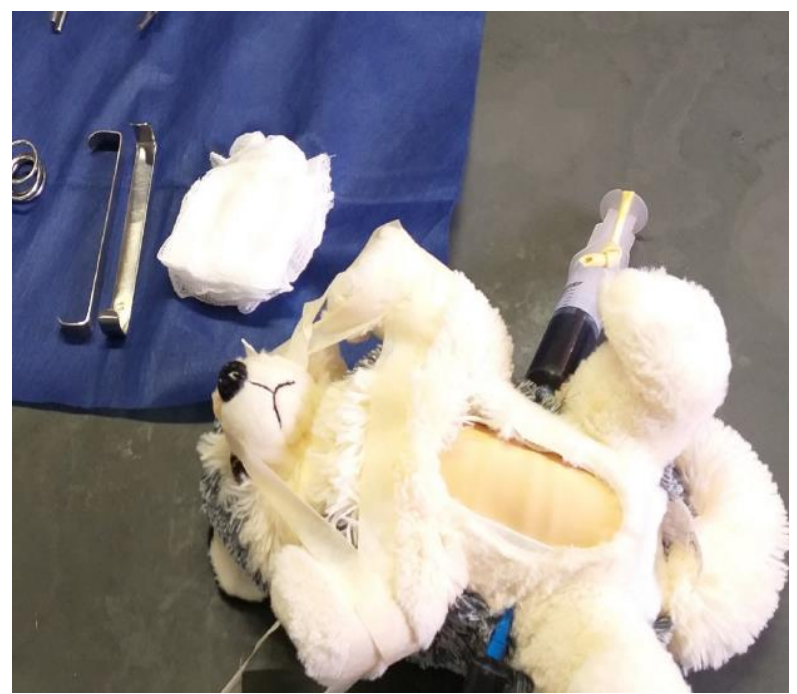

Figura 5. Simulador de parede abdominal montado em bichinho de pelúcia, com as seringas preparadas para a simulação da hemorragia, quando incisado.

\section{Percepção dos estudantes ao método de ensino}

Inicialmente, foi obtido Termo de Consentimento Livre e Esclarecido de cada estudante. Foi aplicado um questionário de cinco pontos de Likert (Thaman et al, 2013) a treze estudantes, ao final do semestre. O questionário era composto de nove questões, sendo cinco favoráveis ao método (A-E) e quatro contrárias ao mesmo (FI). As respostas 4 e 5 correspondiam à concordância com a questão, enquanto as respostas 4 e 5 correspondiam à discordância. A resposta 3 era considerada neutra (Tabela 1).

\section{Análise estatística}

A avaliação dos estudantes foi compilada por meio de frequências de respostas e analisada pelos testes Qui-quadrado $\left(\chi^{2}\right)$ e de Fischer, estabelecendo-se nível de significância de 5\%.

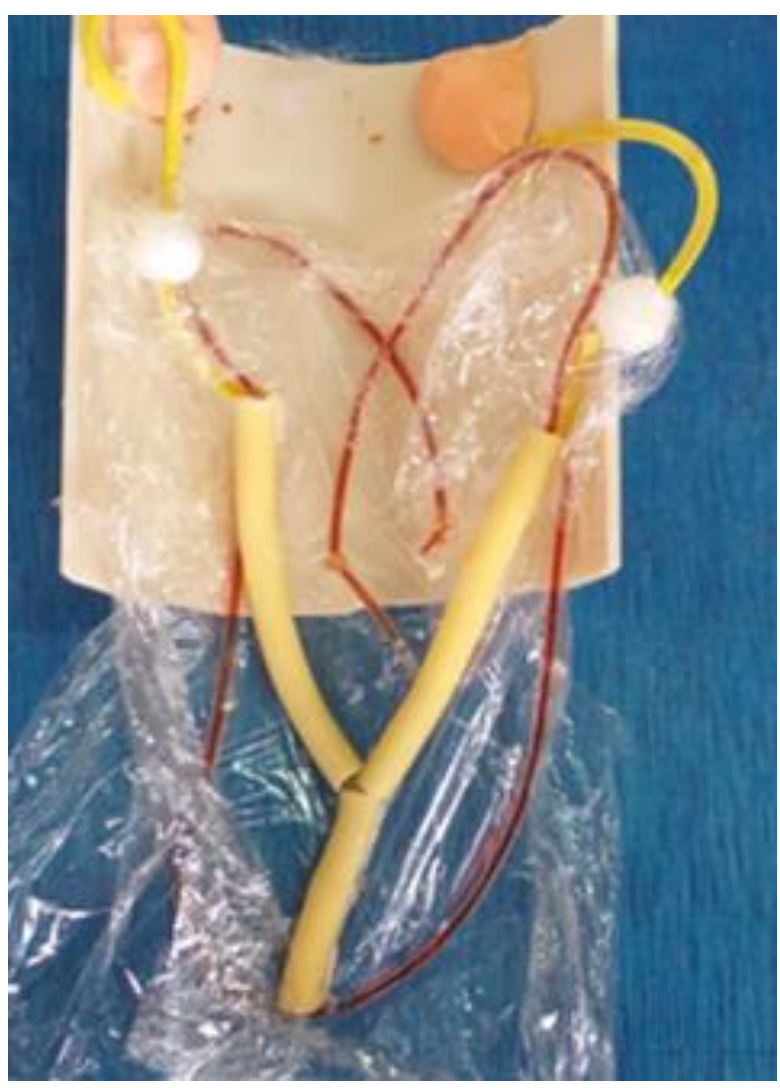

Figura 6. Modelo de útero e ovário, a ser introduzido em bichinho de pelúcia, para treinamento de ovariossalpingohisterectomia (OSH).

Tabela 1. Questionário aplicado aos estudantes para avaliação de sua percepção em relação ao aprendizado das habilidades cirúrgicas pelo método de ensino utilizado.

A. O método foi atrativo.

B. O método foi de fácil compreensão.

C. O método criou interesse pela prática cirúrgica.

D. O método melhorou a compreensão sobre as manobras cirúrgicas.

E. O método tornou o aprendizado mais produtivo.

F. O método requereu muito esforço mental.

G. O método não criou interesse na prática cirúrgica.

H. O método prejudicou a compreensão sobre as manobras cirúrgicas.

I. O método tornou o aprendizado mais difícil.

Respostas:

1 Discordo totalmente, 2 Discordo, 3 Nem discordo nem concordo, 4 Concordo, 5 Concordo totalmente

\section{Resultados}

Após construírem as plataformas e receberem aulas teóricas, os estudantes puderam realizar as manobras cirúrgicas nos diferentes modelos. Todos os dispositivos foram confeccionados manualmente e mostraram-se satisfatórios para as práticas propostas. 
Nos bastidores de EVA e nas bananas acopladas aos suportes, puderam realizar diferentes tipos de incisões, como, segurando o bisturi como um lápis ou como arco de violino, incisões magistrais retas, incisões elípticas, incisões curvilíneas, em "S", elípticas etc. (Figura 2), bem como realizaram diferentes suturas das incisões, em seguida.

Nas plataformas para treinamento de ligaduras vasculares, foram capazes de realizar técnicas de pinçamento, ligaduras circundantes, transfixantes, pediculação e técnica de três pinças (Figura 3), assim como realizaram manobras hemostáticas no simulador de parede abdominal, o qual simulou sangramento substancial após a incisão (Figuras 7 e 8).

Os estudantes também tiveram a oportunidade realizar treinamento prático em ovariohisterectomia no modelo construído, antes da realização da técnica em animais, segundo cada passo da técnica. Neste caso, em particular, alunos que participaram posteriormente da cirurgia em animais vivos, relataram, além da importância do reconhecimento das estruturas e do treinamento, particularmente, a relevância da identificação e da sensação do rompimento do ligamento suspensório no modelo previamente utilizados.

Em relação à percepção dos discentes ao método de ensino adotado, houve elevada frequência de respostas concordantes ao método (respostas 4-5) para questões A-E (favoráveis ao método) e elevada frequência de respostas desconcordantes (respostas 1-2) em relação a questões desfavoráveis ao método (questões F-I) $(\mathrm{p}<0,05$ - Tabela 2).

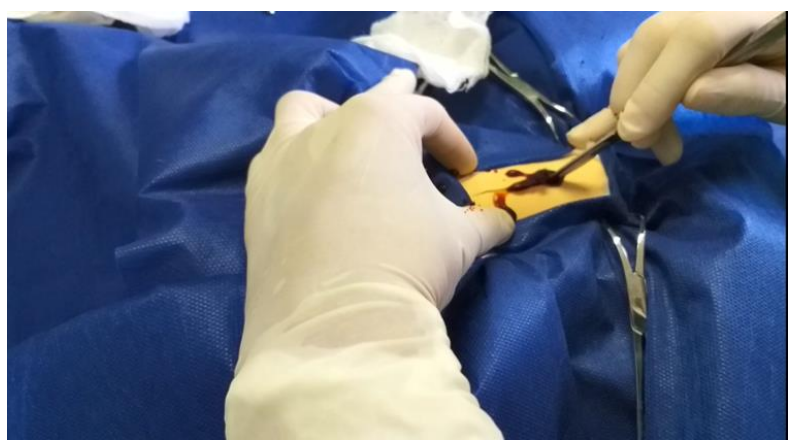

Figura 7. Incisão no simulador da Figura 4, acoplado a abdômen de cãozinho de pelúcia. Notar a simulação do sangramento.

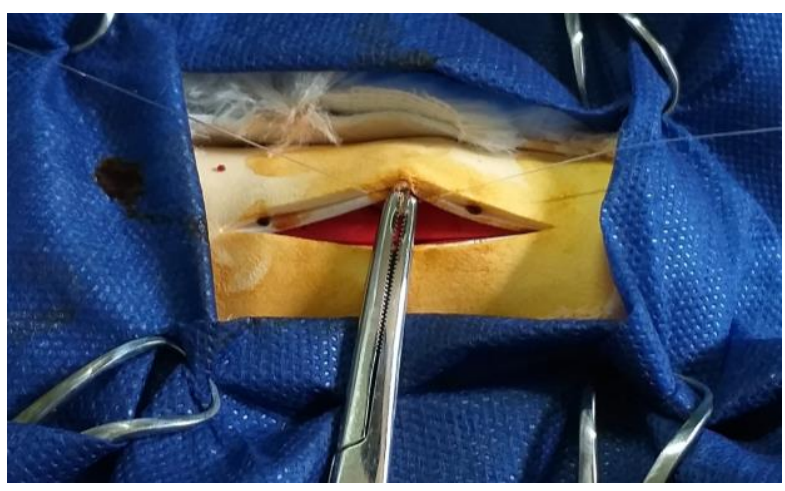

Figura 8. Mesmo modelo da Figura 7, realizando a ligadura.

Tabela 2. Frequências percentuais $(\%)$ de respostas dadas individualmente $(\mathrm{n}=13)$ ao questionário aplicado em relação ao aprendizado das habilidades cirúrgicas pelo método de ensino utilizado.

\begin{tabular}{|c|c|c|c|c|c|c|c|}
\hline \multirow{2}{*}{ Questão } & \multicolumn{5}{|c|}{ Resposta } & \multicolumn{2}{|c|}{$P$} \\
\hline & 1 & 2 & 3 & 4 & 5 & $\chi^{2}$ & Fisher \\
\hline A & $0,00(0)^{\mathrm{cB}}$ & $0,00(0)^{\mathrm{cB}}$ & $0,00(0)^{\mathrm{cB}}$ & $30,77(4)^{\mathrm{bAB}}$ & $69,23(9)^{\mathrm{aA}}$ & $<0,0001$ & $<0,0001$ \\
\hline B & $0,00(0)^{\mathrm{bB}}$ & $0,00(0)^{\mathrm{bB}}$ & $0,00(0)^{\mathrm{bB}}$ & $38,46(5)^{\mathrm{aA}}$ & $61,54(8)^{\mathrm{aA}}$ & $<0,0001$ & $<0,0001$ \\
\hline $\mathrm{C}$ & $0,00(0)^{\mathrm{cC}}$ & $0,00(0)^{\mathrm{cC}}$ & $7,69(1)^{\mathrm{bcBC}}$ & $53,85(7)^{\mathrm{aA}}$ & $38,46(5)^{\mathrm{abAB}}$ & 0,0005 & 0,0005 \\
\hline $\mathrm{D}$ & $0,00(0)^{\mathrm{bB}}$ & $0,00(0)^{\mathrm{bB}}$ & $23,08(3)^{\mathrm{bB}}$ & $7,69(1)^{\mathrm{bB}}$ & $69,23(9)^{\mathrm{aA}}$ & $<0,0001$ & $<0,0001$ \\
\hline $\mathrm{E}$ & $0,00(0)^{\mathrm{bB}}$ & $0,00(0)^{\mathrm{bB}}$ & $0,00(0)^{\mathrm{bB}}$ & $38,46(5)^{\mathrm{aA}}$ & $61,54(8)^{\mathrm{aA}}$ & $<0,0001$ & $<0,0001$ \\
\hline $\mathrm{F}$ & $15,38(2)^{\mathrm{abAB}}$ & $38,46(5)^{\mathrm{aA}}$ & $38,46(5)^{\mathrm{aA}}$ & $7,69(1)^{\mathrm{abAB}}$ & $0,00(0)^{\mathrm{bB}}$ & 0,0373 & 0,0423 \\
\hline G & $61,54(8)^{\mathrm{aA}}$ & $30,77(4)^{\mathrm{abAB}}$ & $7,69(1)^{\mathrm{bcB}}$ & $0,00(0)^{\mathrm{cB}}$ & $0,00(0)^{\mathrm{cB}}$ & 0,0001 & 0,0001 \\
\hline $\mathrm{H}$ & $69,23(9)^{\mathrm{aA}}$ & $30,77(4)^{\mathrm{bAB}}$ & $0,00(0)^{\mathrm{cB}}$ & $0,00(0)^{\mathrm{cB}}$ & $0,00(0)^{\mathrm{cB}}$ & $<0,0001$ & $<0,0001$ \\
\hline I & $84,62(11)^{\mathrm{aA}}$ & $7,69(1)^{\mathrm{bB}}$ & $7,69(1)^{\mathrm{bB}}$ & $0,00(0)^{\mathrm{bB}}$ & $0,00(0)^{\mathrm{bB}}$ & $<0,0001$ & $<0,0001$ \\
\hline
\end{tabular}

a-cFrequências percentuais seguidas por letras minúsculas diferentes na mesma linha diferem significativamente ao nível de significância menor ou igual a 5\% pelo teste de Chi-quadrado. ${ }^{\mathrm{A}-\mathrm{C}}$ Frequências percentuais seguidas por letras maiúsculas diferentes na mesma linha ao nível de significância menor ou igual a 5\% pelo teste de Fisher exato.

\section{Discussão}

O aprendizado de habilidades técnicas é tema extremamente relevante no que tange a educação em cirurgia veterinária e as alternativas ao ensino têm sido objeto de estudos e debates nos últimos anos. O uso de simuladores apresenta algumas vantagens sobre cadáveres animais vivos, forçando os indivíduos a desenvolver suas 
habilidades motoras e elevar sua confiança e eficácia (Smeak, 2006).

Os métodos orientados por habilidades para treinamento em cirurgia focam em uma habilidade fundamental específica e não no procedimento per se, sendo mais vantajosos que os métodos orientados por procedimentos (Smeak, 2007). Diversos modelos têm sido propostos para o treinamento de habilidades cirúrgicas (Smeak, 2007; Fransson et al., 2010; Hill e Smeak., 2010; Costa Neto et al., 2012; Denaday et al., 2014; Giusto et al., 2015; Fahie et al., 2016; Read et al., 2016; Williamson et al., 2019).

Os modelos propostos no presente trabalho foram facilmente confeccionados de maneira artesanal, com material, em sua grande maioria, oriundo de descartes, tendo sido eficiente para o objetivo proposto. Muitas instituições requerem que os estudantes adquiram seu material para treinamentos. Cada aluno pôde confeccionar seus próprios modelos, os quais podiam ser facilmente transportados para qualquer lugar, permitindo assim prática a qualquer momento e local. Os dispositivos poderão ser uma alternativa pedagógica de baixo custo aos estudantes de medicina veterinária no ensino e prática de manobras cirúrgicas básicas. De fato, Denaday et al. (2014) recomendaram alguns modelos de baixa fidelidade (frutas, EVA e plataformas de borracha) para o treinamento de habilidades cirúrgicas básicas, como ferramenta complementar a programas e simuladores disponíveis.

Williamson et al. (2019) compararam dois modelos de simuladores de cavidade abdominal, o DASIE (Dog Abdominal Surrogate for Instructional Exercises), o qual consiste de um cilindro de lâminas de espuma utilizado no treinamento cirúrgico e um modelo similar, porém confeccionado em silicone. Ambos os métodos apresentaram resultados similares em relação à evolução das habilidades cirúrgicas após a prática. Os modelos descritos foram inicialmente utilizados para o treinamento de suturas e não simulavam o sangramento. Nosso modelo de simulador de parede abdominal permite não apenas $o$ treinamento de suturas, mas também de manobras de hemostasia, além do preparo o campo operatório e incisão do modelo, uma vez que é acoplado ao correspondente do abdômen de um bichinho de pelúcia.

Bananas têm sido usadas no treinamento de habilidades cirúrgica há muito tempo. Foi desenvolvida uma plataforma simples e barata para a fixação de bananas, para treinamento de incisões e suturas. A superfície côncava das bananas pôde se encaixar perfeitamente na superfície convexa do suporte, tornando as manobras mais precisas. Os estudantes podiam praticar incisões na casca, sem atingir a fruta, treinando a destreza e precisão cirúrgica.

Griffon et al. (2000) descreveram um modelo de trato reprodutor feminino, com simulação de sangramento, que se mostrou efetivo no treinamento de ovariohisterectomia. O modelo apresentado no presente trabalho foi prático, de fácil utilização por parte dos estudantes, encaixando-se no interior do correspondente à cavidade abdominal de bichinhos de pelúcia e permitiu a realização da técnica em todas suas etapas, com simulação da ruptura do ligamento suspensório, inclusive. Entretanto, não possibilitava a simulação de sangramento, apesar de se destacarem as estruturas correspondentes à vasculatura ovariana e uterina (sondas preenchidas por hidróxido férrico). Da mesma forma, futuramente poder-se-á criar o mesmo sistema de sangramento utilizado para a parede abdominal, no trato reprodutivo.

A utilização de bichinhos e pelúcia permitiu aos estudantes a prática de várias etapas da cena cirúrgica, como o posicionamento na mesa, a antissepsia do campo cirúrgico e a colocação dos panos de campo. Os bichinhos foram muito bem aceitos, uma vez que possuem similaridade com os futuros pacientes e um carisma natural, por se tratar de brinquedos infantis. Tanto os simuladores de parede abdominal quanto os do aparelho genital feminino eram acoplados no interior dos brinquedos, permitindo uma situação bastante realística, sendo as peças substituídas, como um refil.

\section{Conclusão}

De acordo com a percepção dos estudantes, nossos modelos foram um método atrativo e de fácil entendimento, despertaram interesse na prática cirúrgica, melhoraram a compreensão a respeito das manobras e tornaram o aprendizado mais produtivo para o ensino e treinamento de habilidades cirúrgicas, provendo treinamento complementar de modo alternativo.

\section{Conflito de interesse}

Os autores declaram não existir conflito de interesse. 


\section{Comitê de Ética}

O estudo foi submetido à Comissão de Ética em Pesquisa do Conselho Nacional de Saúde (protocolo $\mathrm{n}^{\circ}$ CAAE 39318720.0.0000.5108).

\section{Referências}

Costa Neto, J.M.; Martins Filho, E.F; Gomes Jr.; D.C, Moraes, V.J.; Teixeira D. M.; Silva J. J.; Silva, V.S.C.; Resende L.S. Bastidor aplicado ao ensino da técnica cirúrgica veterinária síntese dos tecidos. Medvep, 10(32): 16-21, 2012.

Denaday, R.; Saad-Hossne, R.; Toledo, A.P.; Kirylko, L.; Souto, L.R.M. Low-fidelity bench models for basic surgical skills training during undergraduate medical education. Revista do Colégio Brasileiro de Cirurgiões, 41(2): 13745, 2014.

Fahie, M.; Cloke, A.; Lagman, M.; Levi, O.; Schmidt, P. Training veterinary students to perform ovariectomy: using the MOOSE spay model with traditional method versus the dowling spay retractor. Journal of Veterinary Medical Education, 43(2): 176-83, 2016.

Fransson, B.A.; Ragle, C.A. Assessment of laparoscopic skills before and after simulation training with a canine abdominal model. Journal of American Veterinary Medical Association, 236(10): 1079-84, 2010.

Giusto, G.; Comino, F.; Gandini, M. Validation of an effective, easy-to-make hemostasis simulator. Journal of American Veterinary Medical Association, 42(1): 85-8, 2015.

Griffon, D.J.; Cronin, P.; Kirby, B. Veterinary surgery. Evaluation of a hemostasis model for teaching ovariohysterectomy in veterinary surgery. Veterinary Surgery, 29: 309-316, 2000.

Hill, L.N.; Smeak, D.D. Suspensory ligament rupture technique during ovariohysterectomy in small animals. Compendium: Continuing Education for veterinarians, 32(6): E1-E7, 2010.

Read, E.K.; Vallevand, A.; Farrell, R.M. Evaluation of veterinary student surgical skills preparation for ovariohysterectomy using simulators: a pilot study. Journal of American Veterinary Medical Association, 43(2): 190213, 2016.

Silva, G.R.M.; Matera, J.M.; Ribeiro, A.A. Preservation of cadavers for surgical technique training. Veterinary Surgery, 33: 606-608, 2004.

Smeak, D.D. Ethical surgery training for veterinary students. In: Jukes, N.; Chiuia, M. From guinea pig to computer mouse. $2^{\text {nd }}$ ed. Leicester: InterNICHE, 2006. part B, cap.6, 117-124p.

Smeak, D.D. Teaching surgery to the veterinary novice: the Ohio State University experience. Journal of American Veterinary Medical Association, (5): 620-627, 2007.

Thaman, R.; Dhillon, S.; Saggar, S.S.; Gupta M.; Kaur, H. Promoting active learning in respiratory physiology-positive student perception and improved outcomes. National Journal of Physiology Pharmacy and Pharmacology, 3 (1): 27-34, 2013.

Williamson, J.A.; Brisson, B.A.; Anderson, S.L.; Farrell, R.M.; Spangler, D. Comparison of 2 canine celiotomy closure models for training novice veterinary students. Veterinary Surgery, 48(6): 966-974, 2019. 